\title{
Vertical Distribution of the Plant-Parasitic Nematode, Pratylenchus penetrans, Under Four Field Crops
}

\author{
Mahesh P. Pudasaini, Cornelia H. Schomaker, Thomas H. Been, and Maurice Moens
}

First and fourth authors: Agricultural Research Centre, Department of Crop Protection, Burg. Van Gansberghelaan 96, 9820 Merelbeke, Belgium and Faculty of Bioscience Engineering, Ghent University, Coupure 653, 9000 Ghent, Belgium; and second and third authors: Plant Research International BV, PB 16, 6700 AA, Wageningen, The Netherlands. Accepted for publication 26 October 2005.

\begin{abstract}
Pudasaini, M. P., Schomaker, C. H., Been, T. H., and Moens, M. 2006. Vertical distribution of the plant-parasitic nematode, Pratylenchus penetrans, under four field crops. Phytopathology 96:226-233.

The vertical distribution of Pratylenchus penetrans was monitored in four fields cropped with maize, black salsify, carrot, or potato. Soil samples were collected at 21-day intervals from May 2002 until April 2003 from five plots $\left(2 \times 5 \mathrm{~m}^{2}\right)$ per field. Per plot, 15 cores were taken to a depth of $70 \mathrm{~cm}$ and split into seven segments of $10 \mathrm{~cm}$ each. Within the plots, segments from corresponding depths were pooled. After mixing, 200-g subsamples were taken and nematodes were extracted by zonal centrifugation from the root fraction and the mineral soil fraction separately. In most crops, the root fraction contained more than $50 \%$ of the

total number of P. penetrans. Because the ratio between the numbers of nematodes in the root fraction and mineral soil fraction changes during the growing season, numbers of $P$. penetrans found in the mineral soil fraction cannot be used to estimate the total number in the soil. Therefore, both fractions have to be processed to obtain a reliable estimate of the density. No nematodes were recovered below $50 \mathrm{~cm}$ soil depth, except in the maize field where nematodes were found at $70 \mathrm{~cm}$. The optimum sampling depth for maize, black salsify, carrot, and potato was 45, 25, 25, and $35 \mathrm{~cm}$, respectively. The percentage of nematodes per soil layer was independent of the sampling date, indicating that a defined optimum sampling depth will be applicable throughout all seasons. The cumulative vertical distribution, modeled with a logistic equation, can be used to estimate the sampling error when samples are collected at different depths.
\end{abstract}

The obligately parasitic, root-lesion nematode, Pratylenchus penetrans, has a wide range of hosts and occurs throughout temperate regions $(7,15)$. Under favorable conditions, such as sandy or sandy loam soil (30), P. penetrans can reduce crop yield and quality $(25,28)$. In Belgium, potato, maize, and vegetables frequently suffer from $P$. penetrans infections (N. Viaene, personal communication).

Although $P$. penetrans is often controlled by nematicides (13, 24,26 ), increasing concerns about the environment, food safety, and public health are leading to a gradual ban of most of the currently used nematicides $(18,32)$. One alternative to chemical control is the use of a knowledge-intensive Integrated Nematode Management (INM) system as described by Been and Schomaker (4) for potato cyst nematodes. Because the preplant population density $(\mathrm{Pi})$ of tylenchid nematodes is a predictor for damage $(1,21,28)$, data from soil samples can provide the information needed to guide nematode management. Obviously, soil sampling for estimating nematode densities is the cornerstone of all INM systems. To develop adequate sampling procedures, knowledge is required about the horizontal and the vertical distributions of nematodes and how these distributions change in time. Knowledge of the vertical distribution will help determine the sampling depth needed to obtain a useful estimate of nematode population density. Information on changes of the vertical distribution over time will indicate whether sampling depth should change with time of year or crop phenology.

When studying the vertical distribution of Pratylenchus spp. in an apple orchard, Ogiga and Estey (22) found these nematodes as

Corresponding author: M. Moens; E-mail address: m.moens@clo.fgov.be

DOI: $10.1094 /$ PHYTO-96-0226

(C) 2006 The American Phytopathological Society deep as $80 \mathrm{~cm}$; the highest densities were between 20 and $40 \mathrm{~cm}$. In a soybean field, Brodie (5) detected the highest densities of $P$. brachyurus between depths of 45 and $75 \mathrm{~cm}$. Sohlenius and Sandor (29) measured population densities of Pratylenchus spp. under grass and barley and found the highest densities at 20 to $30 \mathrm{~cm}$. After monitoring $P$. penetrans at 2 -week intervals during 2 years under raspberry, Forge et al. (10) reported no consistent correlation between the vertical distribution of the nematode and the distribution of host roots. Little information is available on the vertical distribution of $P$. penetrans in annual crops, and nothing is known about its distribution under crops in Belgium.

$P$. penetrans is found both in the mineral soil fraction and in the root fraction during the full duration of crop growth $(10,19,23)$. Between successive crops, it may overwinter in root remnants $(8,12)$. As a consequence, an accurate estimate of nematode density requires that nematodes be counted in both fractions; nematode density can be severely underestimated if the root fraction is omitted. If a constant relationship between numbers of nematodes in both fractions occurs at any time during the crop growth and between successive crops, the actual nematode density could be derived from data obtained from one of the fractions. Merrifield and Ingham (19) reported a rapid change over time in the proportion of $P$. penetrans population in the root and mineral soil fractions for perennial crops. No such information is available for annual crops.

To improve sampling schemes for estimating $P$. penetrans densities in field-grown crops, we investigated the distribution of this nematode in both the mineral and root fraction of the soil at different depths and during a full year. The specific objectives were to (i) quantify and model the vertical distribution of $P$. penetrans; (ii) determine the optimal sampling depth for an unbiased estimation of the nematode density for a number of crops; (iii) examine the importance of nematode numbers in the root fraction; and (iv) investigate the existence of a constant ratio between the 
population densities in the mineral soil and root fraction in order to omit the processing of the latter.

\section{MATERIALS AND METHODS}

Presampling. Four fields were selected after intensive presampling showed high numbers of monospecific populations of $P$. penetrans. In each of the fields, one experiment with either maize (Zea mays), potato (Solanum tuberosum), carrot (Daucus carrota), or black salsify (Scorzonera hispanica) was established at the area with the highest $P$. penetrans density.

Crops and field characteristics. In each field, either maize, potato, carrot, or black salsify was sown in spring 2002 (Table 1). The first three crops were harvested in fall 2002; black salsify was harvested in early winter 2003 . The maize field had been cultivated continuously with maize for the previous 25 years. Only this field was winter plowed in the first week of November 2002. All fields were plowed in the first or second week of March 2003. Soil texture, organic matter content, and $\mathrm{pH}$ were determined (Table 1). Fertilization, pesticide applications, and irrigation were according to farmers' usual practice.

Experimental design and sampling. In each field, the experimental site $(25 \times 2 \mathrm{~m})$ was divided into five equal plots (replicates). Soil samples were taken every 3 weeks along the plant rows in each plot. At each sampling date, 15 soil cores $(2.5 \mathrm{~cm}$ diameter and $70 \mathrm{~cm}$ deep) were removed from each plot, 3 to $5 \mathrm{~cm}$ apart from the plants in a stratified way. Each core was then divided into seven segments of $10 \mathrm{~cm}$. For each replicate, the 15 segments from each depth were pooled for further processing. Sampling was terminated just before sowing of the next crop.

Sample processing. After determination of the fresh weight, each soil sample was thoroughly mixed and a 200-g subsample was taken. Roots were separated from the mineral soil by washing the subsample through a $250-\mu \mathrm{m}$ sieve; the mineral soil that passed through the sieve was collected in a beaker and is hereafter referred to as the "mineral soil fraction." Roots retained on the sieve along with soil particles larger than $250 \mu \mathrm{m}$ were poured into another 1-liter beaker. Three hundred milliliters of water was added to the second beaker and stirred. After $5 \mathrm{~s}$ without stirring, during which time the soil particles settled to the bottom but the roots remained suspended, the contents were poured onto a $250-\mu \mathrm{m}$ sieve so that the roots but not the soil particles were collected. If present, stems and leaves were removed. This process was repeated until all roots were collected on the sieve. Hereafter we use the term "root fraction" to refer to roots collected on the sieve, including both fresh roots of the current crop and root debris of previous crops. The root fraction was blotted, weighed, and then macerated for $1 \mathrm{~min}$ at high speed with a commercial Warring blender.

Nematodes were extracted separately from the mineral soil fraction and root fraction in an automated zonal centrifugal machine (11). This machine separates nematodes from soil or macerated roots following the principles of conventional extraction by centrifugation, except that the process is fully automated as follows. The nematode suspensions, either with the macerated root fraction or the mineral soil fraction, were subsampled and automatically transferred along with water and $\mathrm{MgSO}_{4}$ solution (density $=1.2$ ) into a rotor. In this rotor, the nematodes were separated from the other components and retained on the interface between the water and the $\mathrm{MgSO}_{4}$ solution. After centrifugation, nematodes were collected in a beaker through the hollow shaft of the rotor. No sieve was used. The extracted $P$. penetrans were counted. Eggs from the root fraction were considered to be $P$. penetrans and were counted because juveniles or adults of other nematode species were never detected in the roots. Eggs extracted from the mineral soil fraction were not counted because the soil fraction likely contained eggs of many different species that cannot be distinguished from $P$. penetrans based on egg morphology. Nematode counts in the root fraction and mineral soil fraction were converted to numbers per unit dry mass of soil samples as determined by oven drying.

Statistical analysis and modeling. To compare population densities between plots, total nematode counts (root fraction + mineral soil fraction) per soil layer were expressed as a percentage of the total number of nematodes in the core. The variances of these percentages were constant and normally distributed (data not shown). To determine whether the numbers of $P$. penetrans could be pooled either over the plots or over the sampling times, a multifactor analysis of variance (ANOVA) (SPSS Inc., Chicago, IL) was carried out using the following model: nematode count $(\%)=$ constant + plot + layer + time $+($ plot $\times$ layer $)+($ plot $\times$ time $)+($ layer $\times$ time $)+$ error, with fixed effects (soil layer, time, and the interaction between layer and time) and random effects (plot, the interaction between layer and plot, and the interaction between plot and time). The relationship between soil depth and the mean cumulative percentage of nematodes was described by a logistic model:

$$
y=\frac{100}{1+e^{-b(d-m)}}
$$

in which $y$ is the cumulative percentage of nematodes at soil depth $d, 100$ is the total cumulative percentage of nematodes over all layers (i.e., 100\%), $b$ is the slope parameter of the curve, and $m$ is the depth of soil where $50 \%$ of cumulative percentage of nematodes was obtained. This model was used for simulations of the sampling errors associated with the sampling of different soil depths.

\section{RESULTS}

Plant growth and development. Plant growth was normal in all fields except in the potato field where the crop suffered from drought at the start of the growing season, probably because of the high percentage of sand (Fig. 1) and the small quantity of rain. Potato emergence was delayed and yield was less than normal (data not shown). Fresh roots were found as deep as $70 \mathrm{~cm}$ under maize and $50 \mathrm{~cm}$ under black salsify, but root growth was limited to the upper $40 \mathrm{~cm}$ under both carrot and potato.

Distribution of nematodes over different soil layers. Because the multifactor ANOVA performed on the nematode counts, expressed as percentages, did not show any significant effect of time (Table 2), we inferred that, during the growing season and following fallow, increases or decreases in population densities of

TABLE 1. Characteristics of fields selected for data collection

\begin{tabular}{|c|c|c|c|c|c|c|c|c|}
\hline \multirow[b]{2}{*}{ Host } & \multirow[b]{2}{*}{ Soil type ${ }^{a}$} & \multirow[b]{2}{*}{$\mathrm{OM}(\%)^{\mathrm{b}}$} & \multirow[b]{2}{*}{$\mathrm{pH}$} & \multirow[b]{2}{*}{ Sowing date } & \multirow[b]{2}{*}{ Harvest date } & \multicolumn{3}{|c|}{ Previous crops } \\
\hline & & & & & & 2001 & 2000 & 1999 \\
\hline Black Salsify & Sandy loam & 3.7 & 6.1 & 17 April 2002 & 23 January 2003 & Maize & Maize & Potato \\
\hline Carrot & Sandy loam & 3.5 & 5.4 & 7 May 2002 & 28 October 2002 & Bean & Potato & Sugar beet \\
\hline Maize & Sandy loam & 3.3 & 6.0 & 20 April 2002 & 24 October 2002 & Maize & Maize & Maize \\
\hline Potato & Loamy sand & 2.7 & 5.8 & 19 April 2002 & 26 October 2002 & Bean & Sugar beet & Onion \\
\hline
\end{tabular}

${ }^{a}$ According to the U.S. Department of Agriculture system of soil particle size and texture classification triangle.

b Organic matter content. 
P. penetrans took place simultaneously in the different soil layers. Also, the effect of plot and the interaction between time and plot were not significant $(P>0.05)$. Therefore, the data could be averaged over the five plots and plotted against soil layer and time (Fig. 2). Based on the calculated $P$ values of the $F$ test, both layer and the interaction between layer and time were significant sources of variation in nematode densities in all crops. The interaction between plot and layer was only significant in maize and potato (Table 2). However, the variance explained by this interaction was very small compared with the variance explained by layer. We therefore concluded that the relative differences in nematode counts did not vary in time, and that the counts could be averaged over time for further analysis. The mean cumulative percentages of nematodes at increasing soil depth averaged over plot and time were described by a logistic model (Fig. 3). The data fit the model very well. Parameter $b$ indicates the steepness of the slope: the higher the value of $b$, the steeper the slope. The value of parameter $b$ was highest in carrot and lowest in maize. Parameter $m$ represents the soil depth of the upper soil layer in which $50 \%$ of the nematodes are found. Higher values of $m$ indicate a deeper distribution of the nematodes, as observed under maize; lower values indicate nematode concentrations in a relatively shallow layer, as observed under carrot (Fig. 3).

Fresh roots were still found 70 and $50 \mathrm{~cm}$ deep under maize and black salsify, respectively. Root growth was limited to the upper $40 \mathrm{~cm}$ layer under both carrot and potato. The presence of $P$. penetrans was closely related to the presence of host roots (Fig. 3 ). The majority of nematodes were found in soil layers colonized by roots. No nematodes or roots were found below $50 \mathrm{~cm}$ under black salsify, carrot, and potato, and only very low numbers of nematodes were found in the 40 to $50 \mathrm{~cm}$ layer under carrot and potato, where no roots were detected. A small percentage of nematodes could still be recovered from a depth of 60 to $70 \mathrm{~cm}$ under maize. In all fields, more than $50 \%$ of the $P$. penetrans were recovered from the layer between 10 and $30 \mathrm{~cm}$ deep.

Distribution of nematodes in root and mineral soil fractions. Over the full year and under black salsify, maize, and potato, most $P$. penetrans nematodes were found in the root fraction (Fig. 4). Under carrot, however, P. penetrans was more abundant in the root fraction only from the end of July through the end of October. The ratio of densities of $P$. penetrans in the root fraction and the mineral soil fraction was highest during September through October under black salsify, during October under maize, and during September under carrot and potato (Fig. 4). This ratio started to decline sharply under black salsify, carrot, and potato before crop harvest while it declined at harvest under maize. The decline in the ratio was mainly due to a sharp decline of $P$. penetrans in the root fraction lasting until the end of October to early November. Thereafter, the nematode decline in the root fraction decreased, and the ratio became more constant (black salsify = 1.5 , maize $=2.0$, potato $=3.5$, and carrot $=0.6$ ). Under all crops except carrot, the ratio of nematodes in the root fraction/mineral soil fraction was smaller at planting than after harvest. Although black salsify was harvested in late January, the pattern of nematode decline in the root fraction under this crop was similar. More than $50 \%$ of the $P$. penetrans could be retrieved from the root fraction (ratio of root fraction to mineral soil fraction $>1$ ) at any time under all crops except carrot.

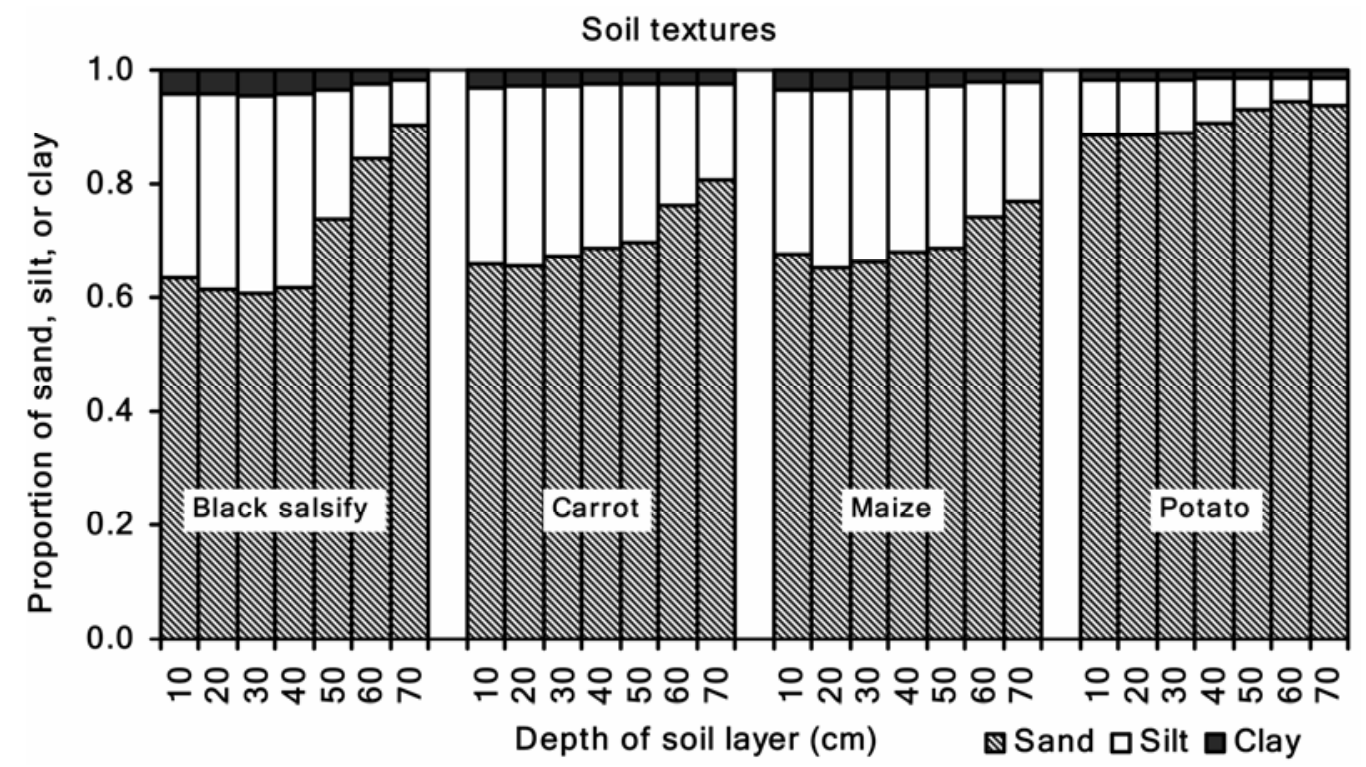

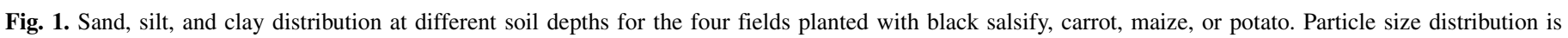
according to the U.S. Department of Agriculture system of soil classification.

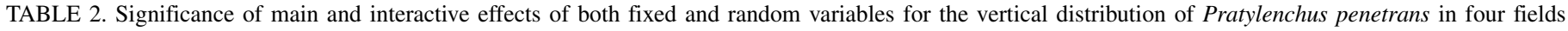
cropped with black salsify, carrot, maize, or potato

\begin{tabular}{|c|c|c|c|c|c|c|c|c|}
\hline \multirow[b]{3}{*}{ Source of variation } & \multicolumn{8}{|c|}{ Crop } \\
\hline & \multicolumn{2}{|c|}{ Black salsify } & \multicolumn{2}{|c|}{ Carrot } & \multicolumn{2}{|c|}{ Maize } & \multicolumn{2}{|c|}{ Potato } \\
\hline & $F$ test & $P$ value & $F$ test & $P$ value & $F$ test & $P$ value & $F$ test & $P$ value \\
\hline Plot & 0.65 & 0.999 & 0.000 & 1.000 & 0.000 & 1.000 & 0.000 & 1.000 \\
\hline Layer & 609.2 & 0.000 & 598.9 & 0.000 & 229.2 & 0.000 & 224.6 & 0.000 \\
\hline Time & 0.711 & 0.764 & 1.463 & 0.149 & 0.487 & 0.938 & 0.599 & 0.854 \\
\hline Plot $\times$ layer & 1.004 & 0.454 & 1.600 & 0.069 & 5.082 & 0.000 & 2.440 & 0.002 \\
\hline Plot $\times$ time & 0.000 & 1.000 & 0.000 & 1.000 & 0.000 & 1.000 & 0.000 & 1.000 \\
\hline Layer $\times$ time & 2.093 & 0.000 & 2.201 & 0.000 & 1.822 & 0.000 & 1.506 & 0.020 \\
\hline
\end{tabular}




\section{DISCUSSION}

Occurrence of $\boldsymbol{P}$. penetrans in the root and mineral soil fractions. As an obligate parasite, $P$. penetrans depends on the availability, both in quality and quantity, of a suitable host. But because of its migratory and endoparasitic behavior, the nematode occurs in both the root fraction and mineral soil fraction of a soil sample. During our observations, the ratio of nematodes in the root fraction and mineral soil fraction increased during the growing season and started to decline before harvest. The increase can be explained by the increasing food availability as the crop develops, whereas the decrease can be explained by slowed growth and senescence of the root system.

During the growing season, many $P$. penetrans were found outside the roots. This might be explained by (i) high initial population densities resulting in competition between the individuals for food, (ii) the ectoparasitic feeding pattern of a proportion of the population $(27,33)$, and (iii) new generations of $P$. penetrans egressing from the root system when food becomes limited in some parts. Except under carrot, the root fraction always contained $50 \%$ or more of the total $P$. penetrans population in soil samples. This agrees with earlier reports. Koen (14) reported a range of 33 to $66 \%$ of $P$. brachyurus located in the root fraction of soil samples taken after harvest of potatoes and during fallow. At least $50 \%$ of a population of $P$. scribneri was reported to overwinter in the root debris of potato and maize between successive growing seasons (16), whereas slightly more than $50 \%$ of the total population of $P$. penetrans was found in the root fraction after harvest of potatoes (8). Obviously, the root fraction is important when estimating $P$. penetrans population densities in soil samples at any time during or after cropping periods. This conclusion was also reached for Meloidogyne chitwoodi by Chen et al. (6).

Because the ratio between the numbers of nematodes in the root fraction and mineral soil fraction changes during the growing season, numbers of $P$. penetrans found in the mineral soil fraction cannot be used to extrapolate the total number in the soil. Although this ratio becomes more or less constant after harvest, it cannot be used when accurate numbers are required because it varies between crops and most probably also between years. Yield prediction based on nematode densities restricted to the mineral soil fraction would certainly be invalid. Therefore, it is necessary to extract nematodes from both fractions as a standard procedure in nematological research and regulatory soil sampling.

Vertical distribution of $\boldsymbol{P}$. penetrans. Previous studies of cropnematode interactions $(5,9,16,17,22,29)$ confirmed that $P$. penetrans was not uniformly distributed through the soil profile. These reports indicated an almost abrupt absence of $P$. penetrans in the layers where roots were absent. In the current study, the population density of $P$. penetrans was highest between 10 and $30 \mathrm{~cm}$ depth, which is similar to that reported for other Pratylenchus species associated with perennial or annual hosts $(17,22,29)$. The host root system is clearly a limiting factor for the vertical distri-
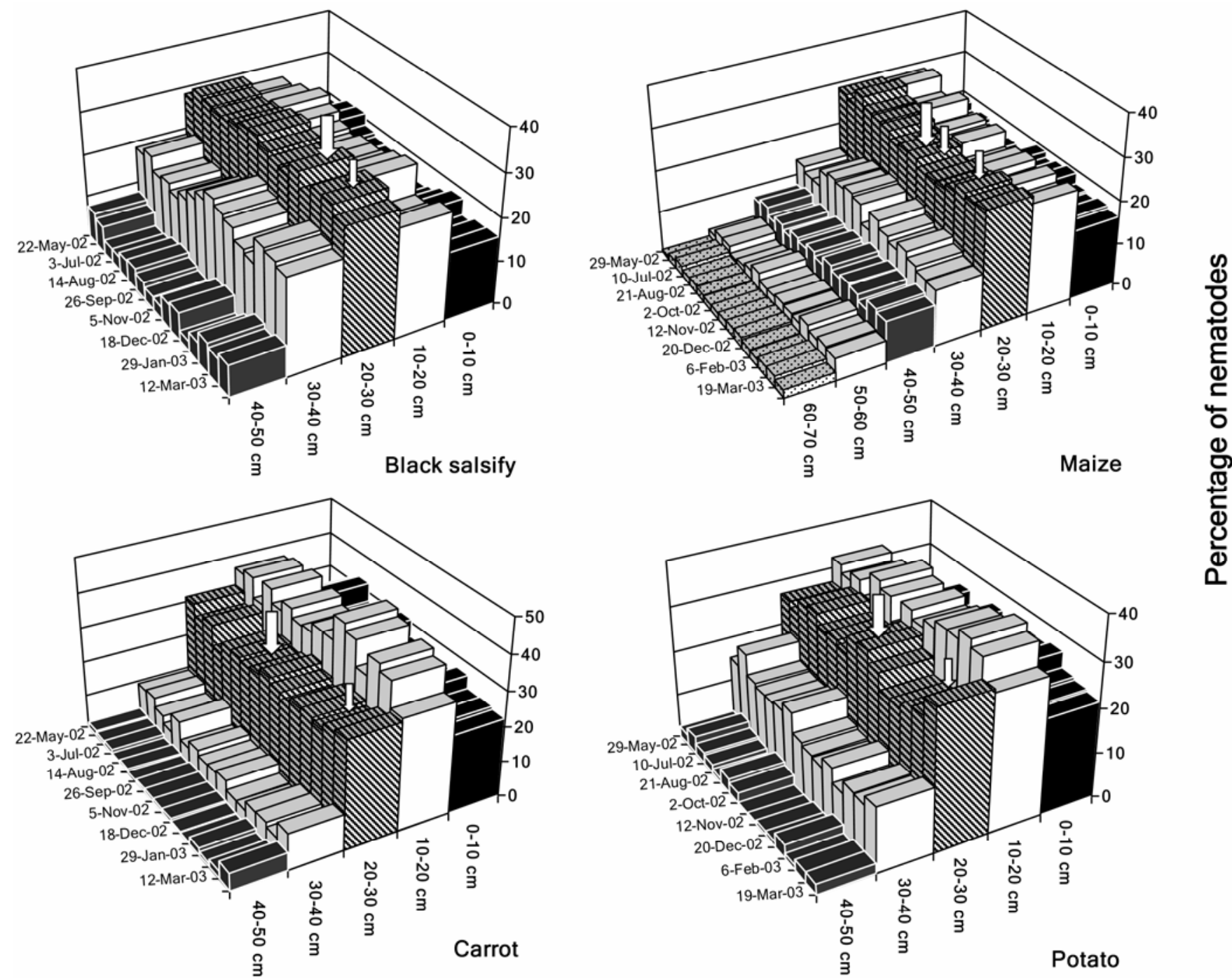

Fig. 2. Percentage of Pratylenchus penetrans in each soil layer throughout the year in four fields planted with black salsify, maize, carrot, or potato. Data were averaged over the five plots per field. The thick arrow indicates harvest date; the thin arrow indicates plowing date (the maize field was plowed twice). 

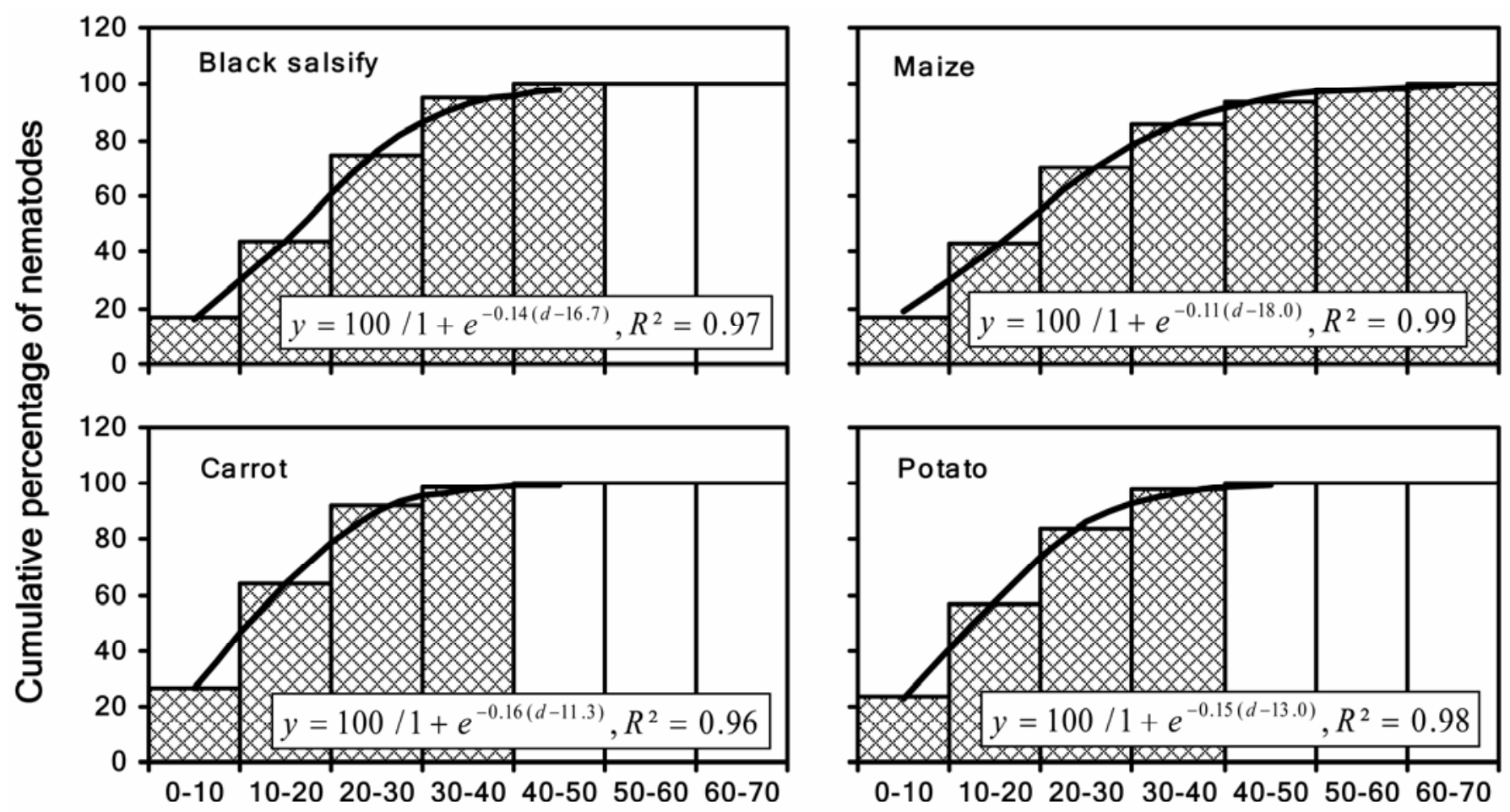

Soil layer $(\mathrm{cm})$

Fig. 3. Cumulative percentage of Pratylenchus penetrans distributed over different soil layers. Data were pooled over plots and times, and means are indicated by bars; filled bars indicate the presence of roots. The solid line represents the logistic model fitted to the cumulative percentages of nematodes present in the soil layers. Explanation of model parameters is described in text $\left(R^{2}\right.$ with $\left.P<0.001\right)$.
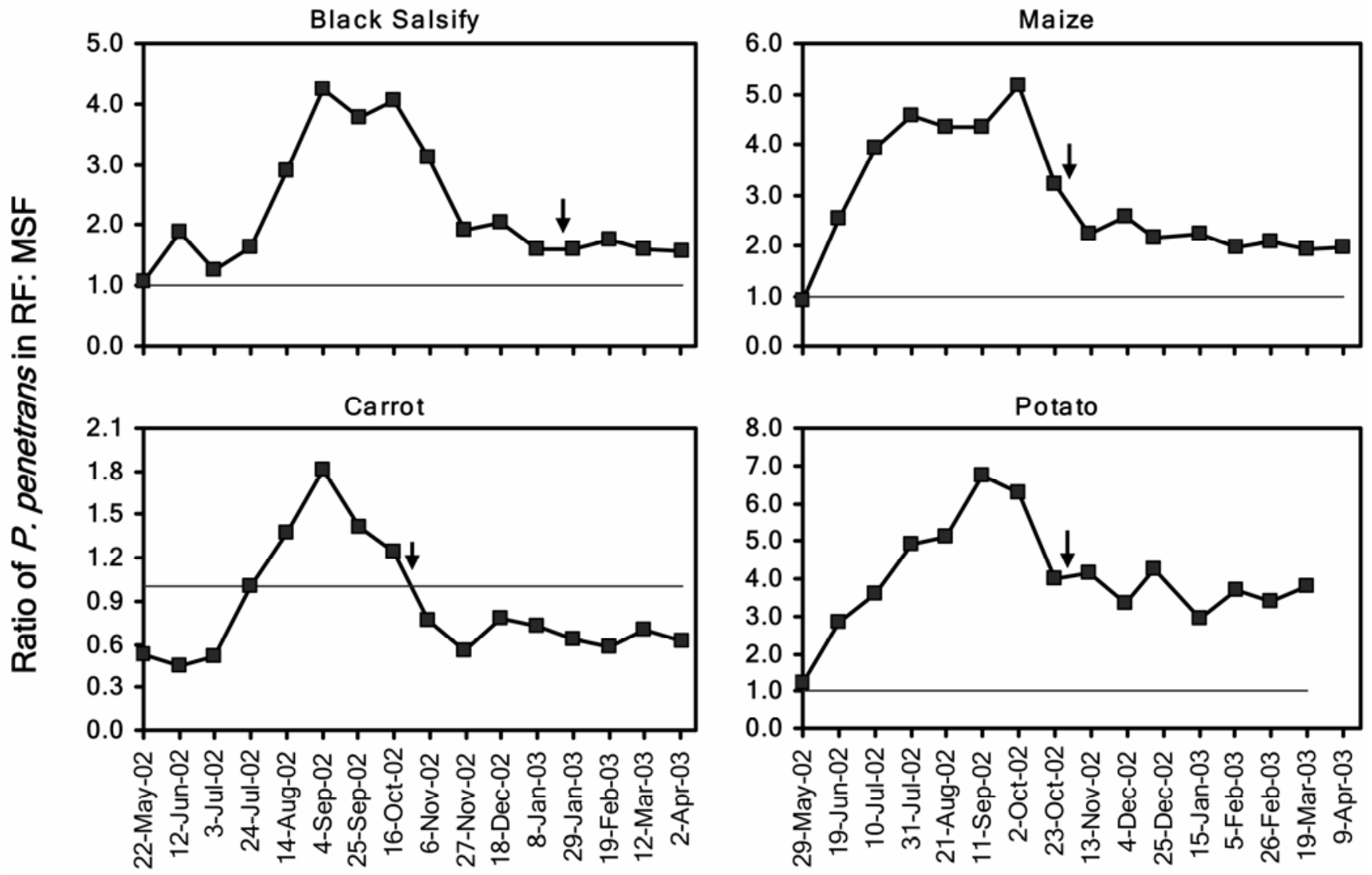

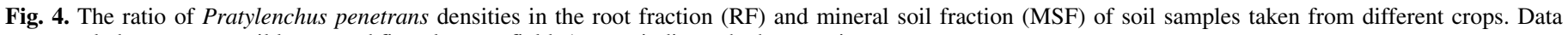
were pooled over seven soil layers and five plots per field. Arrows indicate the harvest time. 
bution of $P$. penetrans. This conclusion was also supported by Ogiga and Estey (22) and Sohlenius and Sandor (29), who demonstrated that the root abundance or root biomass largely determine the vertical distribution of Pratylenchus species. MacGuidwin and Stanger (17) showed that the vertical distribution of P. scribneri was determined by both host root distribution and edaphic conditions. Ferris and McKenry (9) demonstrated that the distribution of Meloidogyne species in and between rows and at greater depths in a vineyard was similar to the distribution of the root system. The distribution of the potato root system was the only factor influencing the presence of potato cyst nematodes in soil layers down to a depth of $80 \mathrm{~cm} \mathrm{(3).}$

In contrast, other workers mentioned that abiotic factors such as soil texture $(5,20)$ or temperature and moisture $(5,14)$ may greatly influence the vertical distribution of nematodes. The soil texture at our experimental sites was similar throughout the whole sampled depth, except in the black salsify field (Fig. 1), and was suitable for $P$. penetrans $(30,31)$. Both moisture and temperature fluctuated through time in the different soil layers (data not shown); however, according to the ANOVA (Table 2), the variation of vertical distribution in time was small compared with the total variance. Both moisture and temperature clearly had only small effects on the vertical distribution. Similarly, no effect of crop harvests and plowing was detected (Fig. 2). Therefore, we conclude that abiotic factors did not greatly affect the vertical distribution of $P$. penetrans.

$P$. penetrans distributions under the crops studied were very well described by the logistic model. The smallest values for the parameter $b$ were found in maize and black salsify, while the largest values were found in potato and carrot. As $b$ and $m$ were negatively correlated $(r=-0.91 ; P<0.001)$, a reverse order was found for $m$, i.e., $m$ values were higher for maize and black salsify and

TABLE 3. Percentage of Pratylenchus penetrans averaged over time in different soil layers under four crops (gray cells depict presence of roots)

\begin{tabular}{lccccccc}
\hline & \multicolumn{6}{c}{ Percentage of nematodes in different soil layers (in cm) } \\
\cline { 2 - 8 } Crop & $0-10$ & $10-20$ & $20-30$ & $30-40$ & $40-50$ & $50-60$ & $60-70$ \\
\hline Maize & 17 & 26 & 27 & 15 & 9 & 4 & 2 \\
Black salsify & 17 & 27 & 31 & 20 & 5 & 0 & 0 \\
Carrot & 27 & 37 & 27 & 7 & 1 & 0 & 0 \\
Potato & 23 & 34 & 27 & 14 & 2 & 0 & 0 \\
\hline
\end{tabular}

smaller for potato and carrot. The small values of the parameter $b$ in maize and black salsify indicate that relative nematode numbers increase more slowly with depth, especially in the upper layers, in these crops than in potato and carrot. Because of this, the soil layer where $50 \%$ of the nematodes are present, represented by the parameter $m$, is shallower for potato and carrot than for maize and black salsify. It is likely that these parameters also reflect root abundance in the different soil layers.

The logistic model can be used to predict population densities and estimate the sampling error when only a part of the vertical distribution is sampled. But because the model cannot be forced through the origin and layer size was $10 \mathrm{~cm}$, the model should not be used to extrapolate the distribution in the first $10 \mathrm{~cm}$ of the soil, where density changes can be expected to be the largest. Predicting sampling error in this layer requires more detailed information on the vertical nematode distribution. Given that the vertical distribution hardly changes in time, this could be achieved with only moderate effort.

Sampling and error. For regulatory soil sampling, labor and cost determine the sampling depth. In view of this, sampling down to $70 \mathrm{~cm}$ depth is not practical. In The Netherlands, soil samples for detection of trichodorids are taken to a depth of $50 \mathrm{~cm}$ after a part of the top soil is removed, and the sample is composed of only a few cores. For ectoparasitic and mobile stages of endoparasitic nematodes, sampling depth is commonly restricted to the upper 25 to $30 \mathrm{~cm}$. In either case, some soil layers are not sampled and, depending on the vertical distribution pattern, the estimation of the population density will be biased. Our results clearly show that the vertical distribution of $P$. penetrans is not uniform but depends on the host. To obtain unbiased density estimates, the optimum sampling depth must be determined for each crop. If sampling to the optimum depth is not feasible, researchers could still obtain an acceptable estimate of nematode density in the root zone if they understood what proportion of the total nematode population was represented in a suboptimum sample.

$P$. penetrans densities are best estimated by sampling the full depth occupied by the host roots (Fig. 3). Sampling only a part of this depth would overestimate the true nematode density (the density in the root zone) if the density in the nonsampled layers was relatively small. Similarly, sampling only a part of the full depth occupied by host roots could underestimate the true nematode density if the density in the nonsampled layers was relatively

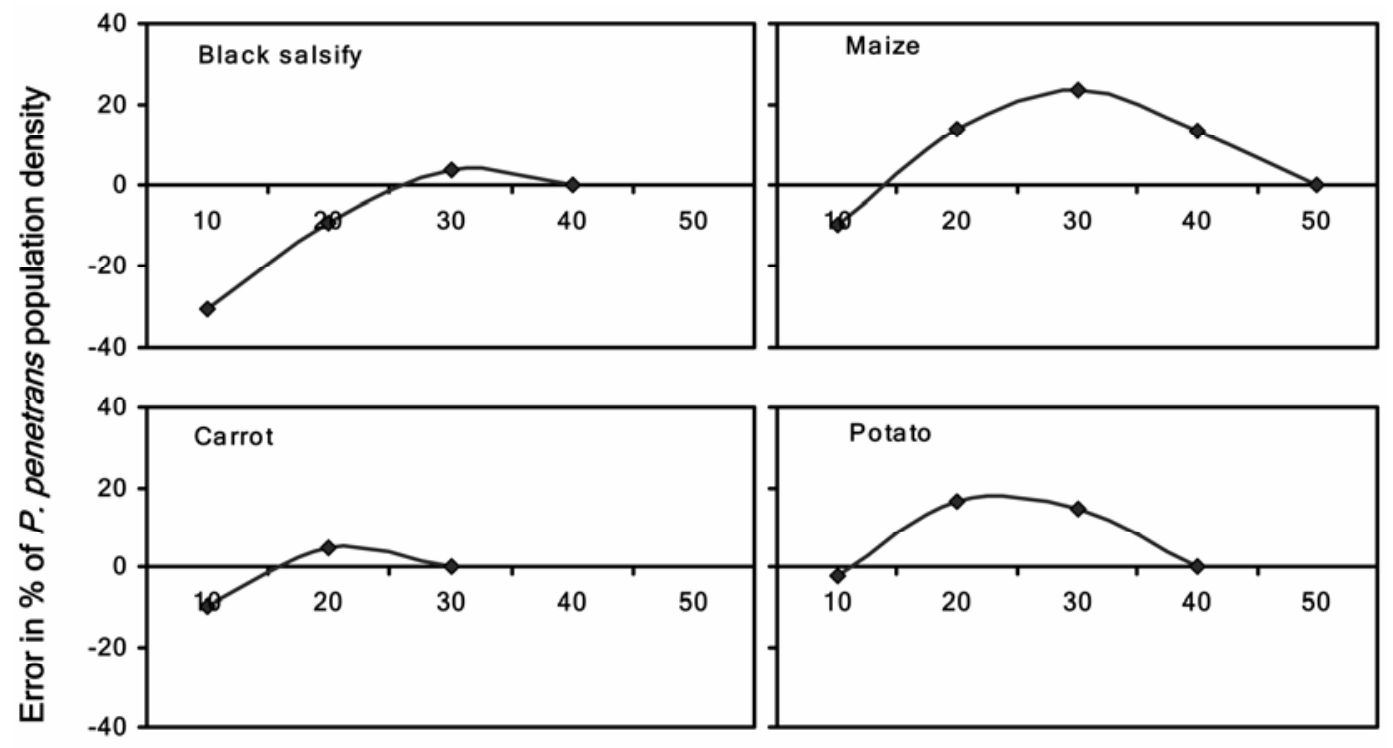

Sampling depth $(\mathrm{cm})$

Fig. 5. Error of the estimation of nematode population density when sampling to different soil depths. 


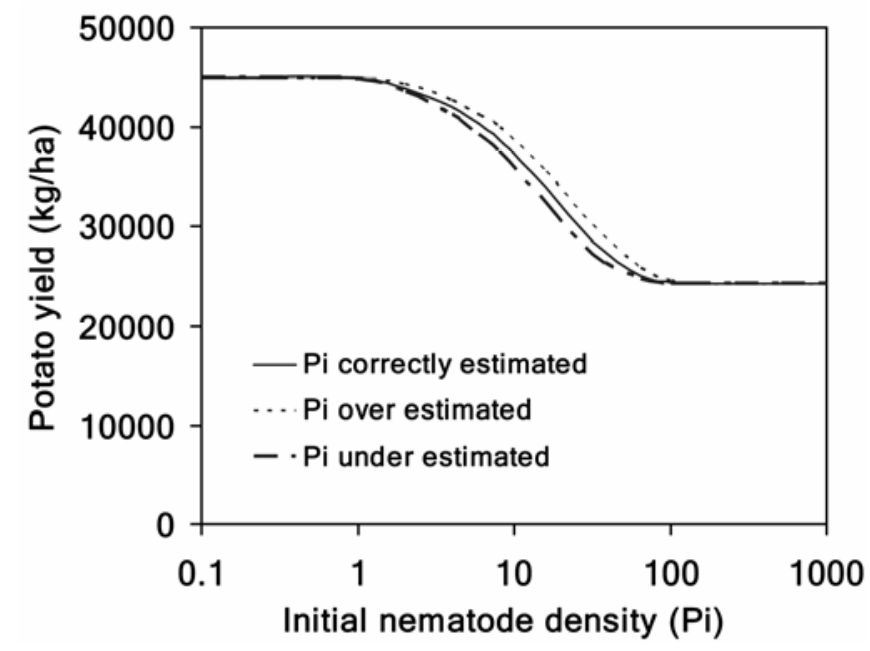

Fig. 6. Damage function curve showing error in the prediction of yield based on a $15 \%$ over- or underestimation of the initial population density of Pratylenchus penetrans ( $\mathrm{Pi}$, nematodes per gram of soil) for potato.

large. Sampling beneath the root zone would always result in underestimation. For example, sampling carrots to $50 \mathrm{~cm}$ rather than to $40 \mathrm{~cm}$ would decrease the estimate of $P$. penetrans population density by $20 \%$, because the 40 to $50 \mathrm{~cm}$ layer is beneath the root zone and contains only $1 \%$ of the total number of nematodes (Table 3 ).

In the following calculations the population density per crop was calculated by including those soil layers that cumulatively contained more than $90 \%$ of the total number of nematodes found. The mean nematode density was calculated at progressing soil depths. At these depths, the over- or underestimation of the true density was calculated (Fig. 5). If an over- or underestimation of $5 \%$ of the true population density is acceptable (a 5\% error should only cause a small error in yield prediction), $25 \mathrm{~cm}$ is an acceptable sampling depth for both black salsify and carrot. For maize and potato, however, accurate yield estimates require sampling to 45 and $35 \mathrm{~cm}$, respectively. Sampling to only $10 \mathrm{~cm}$ would also seem possible for potato and maize but would be risky as this layer is most sensitive to drought. Sampling potato or maize to intermediate depths $(10$ to $30 \mathrm{~cm})$ overestimates nematode density. Although sampling to 40 or even $50 \mathrm{~cm}$ might be feasible for scientific research, it would not be feasible for regulatory and advisory purposes. Moreover, changing the sampling procedure between host plants would be very impractical.

One way to investigate what errors can be tolerated is to link the over- or underestimation of nematode population density to the error of the yield prediction. Based on Seinhorst's equation for yield loss (28) and the parameter values of this equation for $P$. penetrans on potato (tolerance level $[T]=1$ juvenile per gram of soil; minimum yield $[m]=0.54$, as reported by Been and Molendijk [2]), a $15 \%$ error in the estimation of $\mathrm{Pi}$ (initial population density) would result in a density dependent, maximum error of $4 \%$ in the estimation of yield (Fig. 6). It follows that sampling maize and potato to only 25 to $30 \mathrm{~cm}$ should result in useful, if imperfect, estimates of yield loss.

\section{ACKNOWLEDGMENTS}

We thank N. Pudasaini, H. Van De Sype, and E. De Smet for their technical assistance during the collection and extraction of soil samples. N. V. La Corbeille is acknowledged for providing both the funding and the fields used in this investigation. We also thank the anonymous reviewers for their valuable comments.

\section{LITERATURE CITED}

1. Barker, K. R., and Olthof, T. H. A. 1976. Relationship between nematode population densities and crop responses. Annu. Rev. Phytopathol. 14:327353.

2. Been, T. H., and Molendijk, L. P. G. 2003. Aaltjes als opbrengstbeperkende factor in de Fabrieksaardappelteelt. Nota 257, Plant Research International, Wageningen, The Netherlands.

3. Been, T. H., and Schomaker, C. H. 2002. The vertical distribution of potato cyst nematodes (Globodera spp.) in marine-clay and sandy soils in the Netherlands and its implication for statutory soil sampling. (Abstr.) The 54th International Symposium on Crop Protection, University of Ghent, Belgium.

4. Been, T. H., and Schomaker, C. H. 2004. A GEO-referenced decision support system for nematodes in potatoes. Pages 154-167 in: Decision Support Systems in Potato Production; Bringing Models to Practice. D. K. L. MacKerron and A. J. Haverkort, eds. Wageningen Academic Publishers, Wageningen, The Netherlands.

5. Brodie, B. B. 1976. Vertical distribution of three nematode species in relation to certain soil properties. J. Nematol. 8:243-247.

6. Chen, S., Hendrickx, G., and Moens, M. 2000. The importance of organic matter when assaying Meloidogyne chitwoodi soil populations. Russian J. Nematol. 8:147-152.

7. Corbett, D. C. M. 1973. Pratylenchus penetrans. C. H. I. Description of plant-parasitic nematodes. 2, No 25. Commonwealth Institute of Helminthology, St. Albans, Herts, England.

8. Dunn, R. A. 1972. Importance of depth in soil, presence of host roots, and role of eggs as compared to vermiform stages in overwintering of Pratylenchus penetrans at Ithaca, New York. J. Nematol. 4:221-222.

9. Ferris, H., and McKenry, M. V. 1974. Seasonal fluctuations in the spatial distribution of nematode populations in a California vineyard. J. Nematol. 6:203-210.

10. Forge, T. A., DeYoung, R., and Vrain, T. C. 1998. Temporal changes in the vertical distribution of Pratylenchus penetrans under raspberry. J. Nematol. 30:179-183.

11. Hendrickx, G. 1995. An automatic apparatus for extracting free-living nematode stages from soil. (Abstr.) Nematologica 41:308.

12. Kable, P. F., and Mai, W. F. 1968. Overwintering of Pratylenchus penetrans in a sandy loam and a clay loam soil at Ithaca, New York. Nematologica 14:150-152.

13. Kimpinski, J., Arsenault, W. J., and Sturz, A. V. 2001. Differential effect of nematicide treatments on tuber yields in early- and late-maturing potato cultivars. Plant Pathol. 50:509-514.

14. Koen, H. 1967. Note on the host range, ecology and population dynamics of Pratylenchus brachyurus. Nematologica 13:118-124.

15. Loof, P. A. A. 1991. The family Pratylenchidae Thorne, 1949. Pages 363421 in: Manual of Agricultural Nematology. W. R. Nickle, ed. Marcel Dekker, New York.

16. MacGuidwin, A. E. 1989. Distribution of Pratylenchus scribneri between root and soil habitats. J. Nematol. 21:409-415.

17. MacGuidwin, A. E., and Stanger, B. 1991. Changes in the vertical distribution of Pratylenchus scribneri under potato and corn. J. Nematol. 23:73-81.

18. McKenry, M., Buzo, T., Kretsch, J., Kaku, S., Ottomo, E., Ashcroft, R., Lange, A., and Kelley, K. 1994. Soil fumigants provide multiple benefits: Alternatives give mixed results. Calif. Agric. 48:22-28.

19. Merrifield, K. J., and Ingham, R. E. 1996. Population dynamics of Pratylenchus penetrans, Pratylenchus sp., and Criconemella xenoplax on western Oregon peppermint. J. Nematol. 28:557-564.

20. Miller, L. I. 1972. The influence of soil texture on the survival of Belonolaimus longicaudatus. (Abstr.) Phytopathology 62:670-671.

21. Noe, J. P. 1993. Damage function and population changes of Hoplolaimus columbus on cotton and soybean. J. Nematol. 25:440-445.

22. Ogiga, I. R., and Estey, R. H. 1973. Seasonal changes in population density, sex ratios, and vertical distribution of nematodes in orchard soil. Phytoprotection 54:57-68.

23. Olthof, T. H. A. 1971. Seasonal fluctuation in population densities of Pratylenchus penetrans under a rye-tobacco rotation in Ontario. Nematologica 17:453-459.

24. Olthof, T. H. A. 1989. Effects of fumigant and nonfumigant nematicides on Pratylenchus penetrans and yield of potato. J. Nematol. 21:645-649.

25. Olthof, T. H. A., and Potter, J. W. 1973. The relationship between population densities of Pratylenchus penetrans and crop losses in summermaturing vegetables in Ontario. Phytopathology 63:577-582.

26. Philis, J. 1997. Effect of cadusafos and carbofuran against Pratylenchus penetrans and some ectoparasitic nematodes infesting potato in Cyprus. Nematol. Mediterr. 25:169-172.

27. Rebois, R. V., and Huettel, R. M. 1986. Population dynamics, root penetration, and feeding behaviour of Pratylenchus agilis in monoxenic cultures of corn, tomato, and soybean. J. Nematol. 18:392-397.

28. Seinhorst, J. W. 1998. The common relation between population density 
and plant weight in pot and microplot experiments with various nematode plant combinations. Fundam. Appl. Nematol. 5:459-468.

29. Sohlenius, B., and Sandor, A. 1987. Vertical distribution of nematodes in arable soil under grass (Festuca pratensis) and barley (Hordeum distichum). Biol. Fertil. Soil 3:19-25.

30. Townshend, J. L. 1972. Influence of edaphic factors on penetration of corn roots Pratylenchus penetrans and P. minyus in three Ontario soils. Nematologica 18:201-212.

31. Townshend, J. L., and Webber, L. R. 1971. Movement of Pratylenchus penetrans and the moisture characteristics of three Ontario soils. Nematologica 17:47-57.

32. United Nations Environmental Programme (UNEP). 1992. Synthesis report of the methyl bromide interim scientific assessment and methyl bromide interim technology and economic assessment. Page 33 in: Montreal Protocol Assess. Suppl. UNEP, Nairobi, Kenya.

33. Zunke, U. 1990. Ectoparasitic feeding behaviour of the root lesion nematode, Pratylenchus penetrans, on root hairs of different host plants. Rev. Nematol. 13:331-337. 